\title{
Renormalization group approach to the critical behavior of the forest fire model
}

\author{
V. Loreto ${ }^{(1)}$, L. Pietronero ${ }^{(1)}$, A. Vespignani ${ }^{(2)}$ \\ and S. Zapperi ${ }^{(3)}$ \\ 1) Dipartimento di Fisica, Universitá di Roma "La Sapienza" \\ Piazzale Aldo Moro 2, 00185 Roma, Italy \\ 2) Instituut-Lorentz, University of Leiden, P.O. Box 9506 \\ 2300 RA, Leiden, The Netherlands \\ 3) Center for Polymer Studies and Department of Physics \\ Boston University, Boston, MA 02215, USA
}

\begin{abstract}
We introduce a Renormalization scheme for the one and two dimensional Forest-Fire models in order to characterize the nature of the critical state and its scale invariant dynamics. We show the existence of a relevant scaling field associated with a repulsive fixed point. This model is therefore critical in the usual sense because the control parameter has to be tuned to its critical value in order to get criticality. It turns out that this is not just the condition for a time scale separation. The critical exponents are computed analytically and we obtain $\nu=1.0, \tau=1.0$ and $\nu=0.65$, $\tau=1.16$ respectively for the one and two dimensional case, in very good agreement with numerical simulations.
\end{abstract}

PACS numbers: 64.60.Ak, 02.50.-r, 05.40.+j

The Forest-Fire model (FFM) [1,2] has been introduced as a possible realization of Self Organized Criticality (SOC) [3]. The model formulated first by Bak and coworkers [1] contains a tree growth probability $p$ and fire spreading to nearest neighbours. In the limit of very slow tree growth, this model becomes more and more deterministic [4] and shows spiral-shaped fire fronts. For this reason, the original model was modified by Drossel and Schwabl 2] by introducing an ignition parameter $f$, the lightning parameter. This parameter is the probability that during one time step a tree without burning nearest neighbours becomes a burning tree. In this case a critical behaviour, in the sense of anomalous scaling laws, is observed in the double limit $\theta=\frac{f}{p} \rightarrow 0$ and $p \rightarrow 0$. These two limits describe a double separation of time scales: trees grow fast compared to the occurrence of lightning in the system and forest clusters burn down much faster than trees grow. This means that each fire triggered by individual lightning does not overlap with other fires, thus clusters destroyed by fire are well defined objects. The critical state is characterized by a power law distribution $P(s) \sim s^{-\tau}$ of the forest clusters of $s$ sites and the average cluster radius scale as $R \sim \theta^{-\nu}$. Since this critical state is reached independently on the initial conditions and for a wide range of parameter values, it is called self-organized critical state. This statement however is ambiguous in that Self Organized Criticality refers to the tendency of large dynamical systems to evolve spontaneously in a critical state without the fine tuning of any critical parameter. From this point of view, the role played by the parameter $\theta$ is not clear. In fact it seems an effective relevant parameter, as the reduced temperature in thermal phase transitions, in that it allows criticality just for its critical value $\theta=0$.

Usually the limit $\theta \rightarrow 0$ is referred to the existence of a slow driving of the system or, in other words, a time scale separation, also present in the definition of SOC models. It is worth to stress that in the SOC models the requirement of a slow driving of the system is essentially different. In this case, in fact, the time scale separation just affects the lower cut-off of the avalanche size distribution, the upper cut-off being affected by finite-size effects [5]. In the FFM the parameter $\theta$, on the contrary, affects directly the upper cut-off [6] and then it seems to play, in the language of ordinary critical phenomena, the role of a relevant parameter.

In the context of Renormalization Group (RG) methods, a self-organized critical phenomenon can be viewed as a critical phenomenon in which all the parameters are irrelevant, namely the scale invariant dynamics corresponds to an attractive fixed point in the parameter space (phase space). To address the study of intrinsically critical phenomena, we have developed the Fixed Scale Transformation approach to fractal growth and recently we have introduced an RG scheme of novel type to study sandpile models [7,8].

In this letter we follow the same ideas in order to study the FFM including the ignition parameter $f$. This allows us to clarify the role of the critical parameters $\theta$ and $p$ and the nature of the critical state. In fact, we obtain that $\theta$ is the relevant control parameter, so that FFM belongs more to the rich domain of ordinary critical phenomena than to self-organized criticality. In addition we are able to compute analytically the critical exponents characterizing the model in $d=1$ and $d=2$.

For the sake of clarity we recall briefly the dynamical rules of the model we are going to discuss. At each time step the lattice is updated as follows: i) a burning tree becomes an empty site; ii) a green tree becomes a burning tree if at least one of its neighbors is burning; iii) at an empty site a tree can grow with probability $p$; iv) a tree without burning nearest neighbors becomes a 
burning tree with probability $f$.

Starting with arbitrary initial conditions, the system approaches, after a short transient, a steady state the properties of which depend on the parameter values. Let $\rho_{0}, \rho_{1}$ and $\rho_{2}$ be the mean densities of empty sites, of trees and of burning trees in the stationary state. These probabilities describe in full generality the stationary properties of the model, while, as for the dynamical properties, we will refer to a phase space defined by the parameters $p$ and $\theta=\frac{f}{p}$. In such a scheme $p$ and $\theta$ parameterize the dynamics of the system.

We should now extend the characterization of the stationary and dynamical properties of the system at a generic scale $b^{(k)}=b_{0} 2^{k}$ by considering coarse-grained variables $p^{(k)}, f^{(k)}$ and $\rho^{(k)}=\left(\rho_{0}^{(k)}, \rho_{1}^{(k)}, \rho_{2}^{(k)}\right)$. In the following we will mainly refer to the two-dimensional case but all the procedure can be drawn also in the onedimensional case. The stationary properties of coarse grained variables are defined as follows (Fig.1): A cell of size $b^{(k)}$ is considered as "green" if it is spanned from left to right by a connected path of green sites at scale $b^{(k-1)}$. On the opposite a cell is empty if it is not spanned by a connected path of green sites. Finally, we consider a cell as burning if it contains at least one burning tree. In the latter case the spanning condition is not necessary because the fire spreads automatically to nearest neighbors sites. Consequently, the dynamical properties of the coarse grained description of the system will be characterized by the variables $p^{(k)}$ and $\theta^{(k)}=\frac{f^{(k)}}{p^{(k)}}$. The first variable express the probability that an empty cell of size $b^{(k)}$ becomes a green tree. The second one is defined using the probability $f^{(k)}$ that a green cell of size $b^{(k)}$ becomes a burning cell because of the arrival of a lightning event.

We now proceed to define a renormalization transformation for the dynamical variables. We will use a cell-tosite transformation on the square lattice, in which each cell at scale $b$ is formed by four sub-cells at scale $b / 2$. Every cell at the larger scale is then characterized by a number of green and empty sub-cells ranging from one to four. The relative weight of each green configuration is given by the probability to have the corresponding number of green and empty sub-cells

$$
W_{\alpha}(\rho)=n_{\alpha} \rho_{0}^{\alpha} \rho_{1}^{4-\alpha},
$$

where $\alpha$ is the number of empty sub-cells and $n_{\alpha}$ is a normalization factor that takes into account the multiplicity of the configurations. In order to define a renormalization transformation we start with an empty or green cell configuration at scale $b$ and study how it evolves using the dynamical rules of the model. Here we consider a transformation defined by the following rules: i) Every series of tree growth processes at scale $b / 2$ that span an empty cell at scale $b$ in the horizontal direction is renormalized in the growth probability $p$ at scale $b$. ii) Every lightning process at scale $b / 2$ that affects a green cell of scale $b$ contributes to the renormalization of the lightning probability $f$. The spanning rule implies that only tree growth processes extending over the size of the new length scale contribute to the renormalized dynamics. Moreover, it ensures the connectivity properties of the green sites in the renormalization procedure. An example of such a renormalization procedure for the lightning probability is shown in fig.2 for the simplest calculation scheme; i.e. $2 \times 2$ cells with left-right spanning condition.. We have two possible processes depending on whether both or just one green sub-cell is hit by a lightning event. The first case occurs with probability $2 f^{(k)}\left(1-f^{(k)}\right)$, the latter with probability $\left(f^{(k)}\right)^{2}$. By summing these probabilities one obtains, the renormalization equation for the configuration with $\alpha=2$. We can write a renormalization equation for each configuration $\alpha$ corresponding to a green site, and, averaging over all the configurations we obtain:

$$
f^{(k+1)}=\sum_{\alpha=2}^{4} W_{\alpha} f_{\alpha}^{(k+1)}
$$

with

$$
\begin{aligned}
& f_{2}^{(k+1)}=f^{(k)}\left(2-f^{(k)}\right) \\
& f_{3}^{(k+1)}=f^{(k)}\left(3-3 f^{(k)}+\left(f^{(k)}\right)^{2}\right) \\
& f_{4}^{(k+1)}=f^{(k)}\left(4-6 f^{(k)}+4\left(f^{(k)}\right)^{2}-\left(f^{(k)}\right)^{3}\right)
\end{aligned}
$$

In an analogous way we can write the renormalization equations for $p^{(k+1)}$. Let us consider an empty cell at scale $b$ of type $\alpha=2$ (see Fig.3). After an updating step, the cell will become green if at least one of the two empty sub-cells has become green. This occurs with probability $\left(p^{(k)}\right)^{2}$ and $2 p^{(k)}\left(1-p^{(k)}\right)$, corresponding to the fact that both or only one sub-cell become green, respectively. In a similar way one can also write a renormalization equation for each configuration $\alpha$ corresponding to an empty site, and finally averaging over all the configurations we obtain

$$
p^{(k+1)}=\sum_{\alpha=0}^{2} W_{\alpha} p_{\alpha}^{(k+1)}
$$

with

$$
\begin{aligned}
& p_{0}^{(k+1)}=\left(p^{(k)}\right)^{2}\left(2-\left(p^{(k)}\right)^{2}\right) \\
& p_{1}^{(k+1)}=p^{(k)}\left(1+p^{(k)}-\left(p^{(k)}\right)^{2}\right) \\
& p_{2}^{(k+1)}=p^{(k)}\left(2-p^{(k)}\right),
\end{aligned}
$$

From the previous equations it is then straightforward to obtain the renormalization equation $\theta^{(k+1)}=$ $f^{(k+1)} / p^{(k+1)}$. These renormalization equations are not yet complete because the statistical weights $W_{\alpha}$ are function of the the density vector $\rho^{k}$. In fact, in order to describe the stationary critical state it is necessary to couple the dynamics to a stationarity condition that gives the renormalization equations for the density vector. This scheme is similar to that used in [8]. The stationarity condition is obtained from the master equations for the 
density vector in the mean field regime by imposing the asymptotic equilibrium condition $(t \rightarrow \infty)$ [9, 10],

$$
\left\{\begin{array}{l}
\rho_{0}^{(k)}=\left(1-\rho_{1}^{(k)}\right) a^{(k)} / p^{(k)} \\
\rho_{1}^{(k)}=\frac{a^{(k)}}{\theta^{(k)} p^{(k)}+4 \cdot a^{(k)}-a^{(k)} \cdot \rho_{1}^{(k)} \cdot(2 d-1)} \\
\rho_{2}^{(k)}=\left(1-\rho_{1}^{(k)}\right) a^{(k)}
\end{array}\right.
$$

where we defined $a^{(k)}=p^{(k)} /\left(1+p^{(k)}\right)$, with the normalization condition $\rho_{0}^{(k)}+\rho_{1}^{(k)}+\rho_{2}^{(k)}=1$. The stationarity condition, summarized in the (5), provides the renormalized density vector at each scale, and it couples the dynamical properties to the stationary ones. It is worth to remark that we do not determine the RG equations for $\rho$ from the coarse graining prescriptions. In fact the stationary properties have to be evaluated considering the average over many dynamical processes. Thus the densities $\rho$ are determined from the renormalized dynamical description of the system, namely eq.s (6) with renormalized parameters. Note also that the RG equations are written in the hypothesis of a double time scale separation: fire spreading and trees growth are not interacting. This is expressed by the consistency relation $p<1 / T(\xi)$, where $T(\xi)$ is the average time needed to burn a cluster of characteristic size $\xi$. Since $T(\xi) \sim \xi^{z}$ where $z$ is the dynamical exponent, and $\xi \sim(f / p)^{-\nu}$ we have that $T(\xi) \sim(f / p)^{-\nu^{\prime}}$ with $\nu^{\prime}=\nu z$. Our RG approach is then suitable to describe what happens in the region of the phase space $(p, \theta)$ defined by the condition $p<\theta^{-\nu^{\prime}}$.

Given this scheme, we can find the fixed points of the renormalization transformation by studying the flow diagram in the phase space of the parameters $(\rho, p, \theta)$. In two dimensions the RG equations show the fixed point $p^{*}=0, \theta^{*}=0$ and $\rho^{*}=(2 / 3,1 / 3,0)$. A complete characterization of the fixed point is obtained by the linearized RG equations around $p^{*}, \theta^{*}$ and $\rho^{*}$. We find only one relevant scaling field that corresponds to an eigenvalue greater than 1 , which is given by

$$
\lambda=\left.\frac{d \theta^{(k+1)}}{d \theta^{(k)}}\right|_{p^{*}, \theta^{*}, \rho^{*}}
$$

Therefore, we have that the fixed point is repulsive in the $\theta$ direction, which of course defines the relevant control parameter. Since the fixed point is repulsive we can determine the exponent of the clusters characteristic length by using the largest eigenvalue, i.e., $\nu=\log 2 / \log \lambda$. We obtain $\nu=0.73$ by using the simple $2 \times 2$ cell renormalization scheme. We can easily improve the results with a $3 \times 3$ cell calculation, which gives $\nu=0.65$, showing that the numerical result converges to the right value with refined renormalization scheme. The exponent $\nu$ describes the divergence of the correlation length by $R \sim \theta^{-\nu}$ and the value obtained is in good agreement with the value $\nu \simeq 0.58$ measured in 6.11]. In this perspective the parameter $\theta=\frac{f}{p}$ plays the role of the relevant critical parameter as the reduced temperature in the thermal phase transitions. For each value of $\theta$, small but finite, the system shows an upper characteristic length in the cluster distribution. Only for $\theta^{*}=0$ the system is critical and shows an infinite correlation length.

The exponent $\tau$ describing the distribution of fire spreading can be obtained as follows. The fires are represented by the clusters of connected sites interested by a burning process. As in [8] we define $K$ as the probability that an active relaxation process (i.e. fire) is limited between the scales $b^{(k-1)}$ and $b^{(k)}$ and it does not extend further:

$$
K=\int_{b^{(k-1)}}^{b^{(k)}} P(r) d r / \int_{b^{(k-1)}}^{\infty} P(r) d r
$$

where $P(r) d r$ is the probability to have a burning cluster with radius between $r$ and $r+d r$. In two dimensions with simple scaling arguments we can conclude that, if $P(s) \sim s^{-\tau}$ and $s \sim r^{2}$ (compact clusters), then $P(r) \sim r^{1-2 \tau}$. Inserting this expression in eq. (8) we obtain (in $d=2) \tau=1-(\log (1-K)) /(2 \log 2)$. In our case $K$ is the probability that at a generic scale $(k)$ all the nearest neighbors of a burning tree are empty and then $K=\left(1-\rho_{1}^{(k)}\right)^{4}$. In the scale invariant regime $\left(\rho_{1}^{(k)}=\rho_{1}^{*}\right), K=0.1975$ and then we obtain for $\tau$ the value $\tau \simeq 1.16$ in excellent agreement with very accurate simulations performed by P. Grassberger [6].

Along the same lines it is possible to compute also the dynamical exponent $z$, and then from scaling laws [11] the remaining critical exponents that characterize the FFM model [12]. Our approach can be naturally extended to the one dimensional forest fire. We can follow the scheme used previously, and along the same lines it is possible to compute the exponent $\nu=1.0$ and $\tau=1.0$ which recover the exact results for the one dimensional case obtained in 13]. We summarize our results for the one and two dimensional case in Table 1 . Details of the one dimensional calculation as well as the two dimensional case will be reported in Ref. [12].

In conclusion, we introduce a novel RG approach suited to study the critical state of the Forest-Fire model. We identify the structure of the phase space in which the RG transformation is constructed and then we introduce the coupling of the renormalization equations with the asymptotic stationarity condition of the systems. By studying the evolution of the system under scale change, we stress the existence of a relevant parameter, $\theta$, namely corresponding to a repulsive fixed point for the RG equations. In this sense, $\theta$ is the critical parameter of the model, and the critical state is reached only with a fine tuning of $\theta$ to its critical value. The existence of this parameter, given by the ratio between the driving rate of the system $f$ and the trees growth rate $p$, places the FFM in the field of ordinary critical phenomena. In fact, it is worth to stress that in sandpile models the same RG analysis [8] shows that no relevant parameter is present, namely the fixed point is completely attractive, and the slow-driving of the system does not affect the infinite 
correlation properties but only the lower cut-off of distributions.

It is a pleasure to thank B. Drossel and P.Bak for interesting discussions.

[1] P.Bak, K. Chen and C. Tang, Phys. Lett. A 147, 297 (1990).

[2] B. Drossel and F. Schwabl, Phys. Rev. Lett.,69, 1629 (1992).

[3] P.Bak, C. Tang and K. Wiesenfeld: Phys.Rev. Lett. 59, 381 (1987); Phys. Rev. A 38, 364 (1988).

[4] P. Grassberger and H. Kantz, J. Stat. Phys.63, 685 (1991); W.K. Moßner, B. Drossel and F. Schwabl, Physica A 190, 205-217 (1992).

[5] R. Cafiero, V. Loreto, L. Pietronero, A. Vespignani and S. Zapperi, Europhys. Lett., 29, 111 (1995).

[6] P. Grassberger, J. Phys. A Math. Gen.26, 2081-2089 (1993).

[7] for a review see: Erzan A., Pietronero L. and Vespignani A.: "The Fixed Scale Transformation approach to fractal growth" subm. to Rev. Mod. Phys.; L.Pietronero, A.Erzan and C. Evertsz, Phys. Rev. Lett. 61, 861 (1988); R. Cafiero, L. Pietronero and A. Vespignani: Phys. Rev. Lett.70, 3939 (1993);

[8] L.Pietronero, A. Vespignani and S. Zapperi, Phys. Rev. Lett. 72, 1690 (1994); A.Vespignani, S.Zapperi and L.Pietronero, Phys. Rev. E 51, 1711 (1995).

[9] B. Drossel and F. Schwabl, Physica A 204, 212-229 (1994).
[10] K. Christensen, H. Flyvbjerg and Z.Olami, Phys. Rev. Lett. 71, 2737 (1993).

[11] S.Clar, B.Drossel and F.Schwabl, Phys. Rev. E 50, 1009 (1994).

[12] V. Loreto, A. Vespignani and S. Zapperi, "Renormalization Scheme for Forest-Fire models", submitted to J. of Phys.

[13] B. Drossel, S. Clar and F Schwabl, Phys. Rev. Lett. 71, 3739 (1993);

\begin{tabular}{|c|c|c|c|}
\hline$d=1$ & $\nu$ & $z$ & $\bar{\tau}$ \\
\hline RG Scheme & 1.0 & 1.0 & 1.0 \\
\hline Exact results $^{+}$ & 1.0 & 1.0 & 1.0 \\
\hline \multicolumn{4}{|l|}{$d=2$} \\
\hline RG Scheme $(2 \times 2)$ & 0.73 & 1.17 & 1.16 \\
\hline RG Scheme $(3 \times 3)$ & 0.65 & 1.02 & 1.16 \\
\hline Simulations* & 0.58 & 1.04 & 1.15 \\
\hline
\end{tabular}

In this table we summarize our results for the critical exponents compared with exact ${ }^{+}$[13] or experimental* results 66,11.

FIGURE CAPTIONS

- Fig.1: Configurations of sites at scale $b^{(k)}$ corresponding to a green cell at scale $b^{(k+1)}$.

- Fig.2: Example of two processes contributing to the renormalization of the lightning probability $f^{(k+1)}$.

- Fig.3: Example of renormalization of the growth parameter $p^{(k+1)}$. 


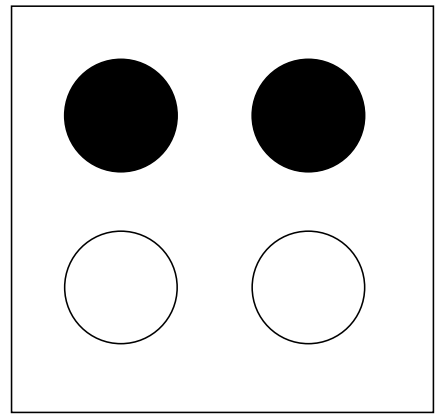

$\alpha=2$

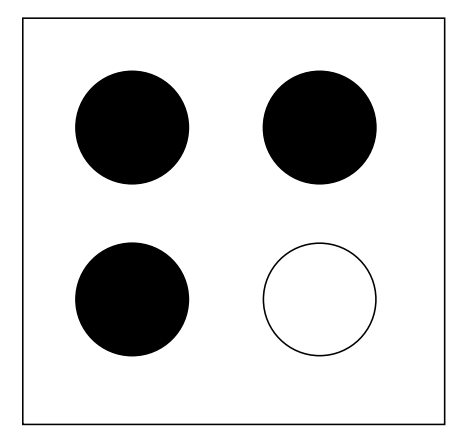

$\alpha=3$

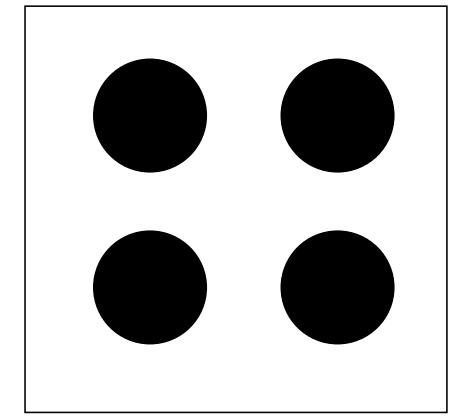

$\alpha=4$ 


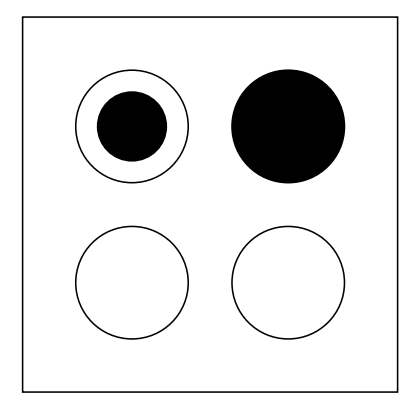

$2 f(1-f)$

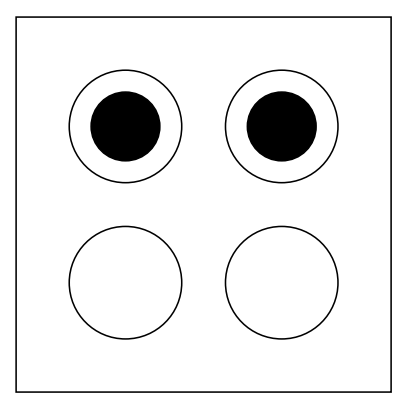

$f^{2}$ 


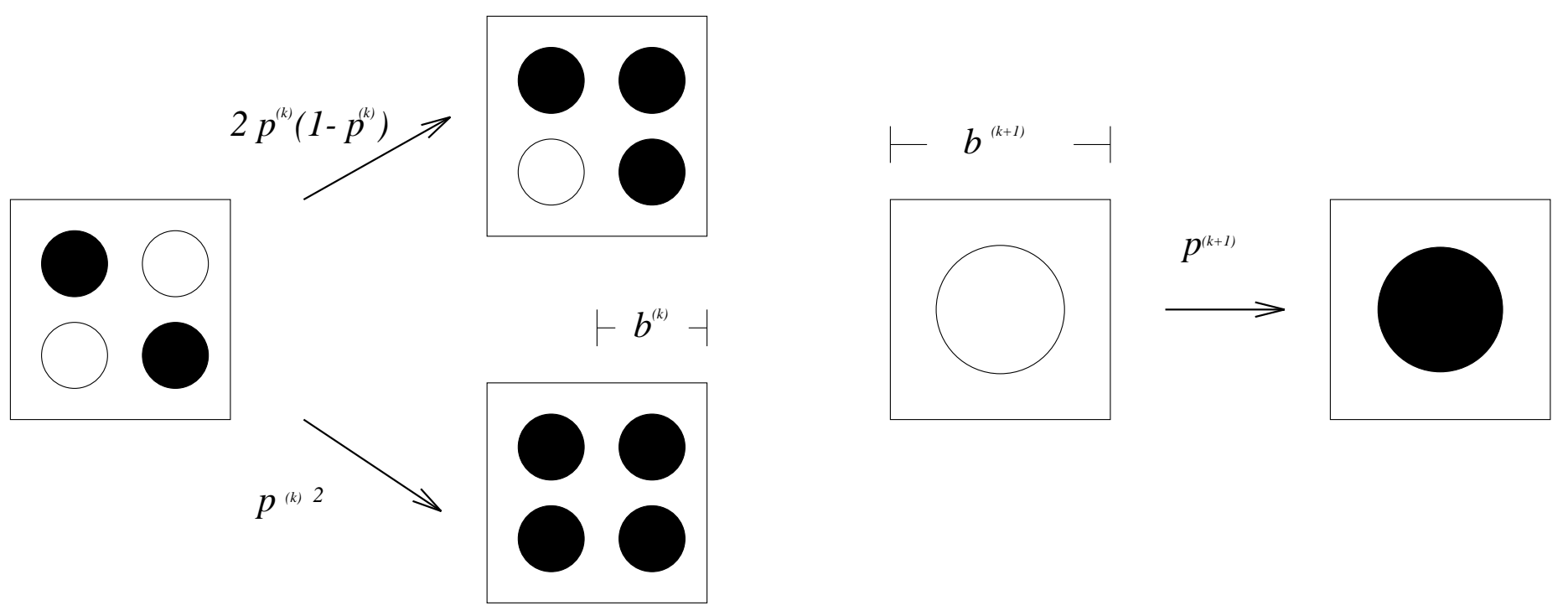

